\title{
The Local Discontinuous Galerkin Method with Generalized Alternating Flux Applied to the Second-Order Wave Equations
}

\author{
Rongpei Zhang $\mathbb{D}^{1}{ }^{1} \mathrm{Jia}_{\text {Liu, }}{ }^{2}$ Shaohua Jiang, ${ }^{3}$ and Di Wang ${ }^{1}$ \\ ${ }^{1}$ School of Mathematics and Systematic Sciences, Shenyang Normal University, Shenyang 110034, China \\ ${ }^{2}$ Department of Foreign Language, Shenyang Normal University, Shenyang 110034, China \\ ${ }^{3}$ School of Art and Design, Shenyang Normal University, Shenyang 110034, China \\ Correspondence should be addressed to Rongpei Zhang; rongpeizhang@163.com
}

Received 12 May 2020; Accepted 20 July 2020; Published 19 August 2020

Academic Editor: Zhile Yang

Copyright (c) 2020 Rongpei Zhang et al. This is an open access article distributed under the Creative Commons Attribution License, which permits unrestricted use, distribution, and reproduction in any medium, provided the original work is properly cited.

\begin{abstract}
In this paper, we propose the local discontinuous Galerkin method based on the generalized alternating numerical flux for solving the one-dimensional second-order wave equation with the periodic boundary conditions. Introducing two auxiliary variables, the second-order equation is rewritten into the first-order equation systems. We prove the stability and energy conservation of this method. By virtue of the generalized Gauss-Radau projection, we can obtain the optimal convergence order in $L^{2}$-norm of $\mathcal{O}\left(h^{k+1}\right)$ with polynomial of degree $k$ and grid size $h$. Numerical experiments are given to verify the theoretical results.
\end{abstract}

\section{Introduction}

The wave equation is an important second-order linear partial differential equation for the description of many traveling wave phenomena, such as the sound waves and seismic waves. Accurate and efficient numerical methods to solve the wave equation are of fundamental importance to the simulation of wave propagation. There are many numerical methods to solve the wave equation, such as the finite element methods (FEMs) $[1,2]$, spectral methods $[3,4]$, and finite volume method [5].

In this paper, we consider the local discontinuous Galerkin (LDG) methods for numerical solution of onedimensional second-order wave equation. The LDG method was first introduced by Cockburn and Shu [6] which was motivated by Bassi and Rebay's work on Navier-Stokes problems [7]. With discontinuous finite element basis function, the LDG method possesses certain flexibility and advantage, such as local (elementwise) conservation, capability of capturing discontinuity, and high accuracy. It can also be easily designed for any order of accuracy since the order of accuracy can be locally determined in each cell.
Many DG methods have been developed for the wave equation in both first-order and second-order forms [2, 8, 9], and some of them are also energy conserving. For instance, in [10], the authors solved the time-dependent acoustic and elastic wave equations by using the discontinuous Galerkin method (DG) for spatial discretization and Crank-Nicolson methods for time integration. And a new class of discontinuous Galerkin (DG) methods for the acoustic wave equation in mixed form is developed and analyzed in [11]. In [9], the new superconvergence results for the local discontinuous Galerkin (LDG) method applied to the secondorder scalar wave equation in one space dimension are presented, and the results show $O\left(h^{p}+1\right)$ convergence rate in $L^{2}$ norm. Chou et al. solved the linear second-order wave equation in heterogeneous media by energy conserving the LDG method [12].

In this paper, we study a new local discontinuous Galerkin (LDG) method for solving the second-order wave equation. We first introduce two auxiliary variables to rewrite the second-order equation as the velocity-stress form of the wave equation. This system is then discretized by the discontinuous Galerkin (DG) method to get the solution of 
the introduced variable. In the design of the LDG method, the numerical flux should be chosen to ensure the stability and high order accuracy. The LDG methods in the previous papers $[9,12]$ applied the purely alternating numerical flux. In this paper, we consider the generalized alternating flux which is motivated by work of Meng et al. [13]. Recently, Cheng et al. [14, 15] developed the LDG method with generalized numerical flux for convection-diffusion equations. They obtained the optimal error estimate based on the construction and analysis of the newly designed generalized Gauss-Radau projections. The local discontinuous Galerkin method based on the generalized alternating numerical flux is also used for solving the one-dimensional nonlinear Burger's equation [16]. By using the generalized Gauss-Radau projection, we can prove that the proposed method can get optimal rate of convergence in $L^{2}$-norm of $\mathcal{O}\left(h^{k+1}\right)$ with polynomial of degree $k$ for wave equation.

After the LDG discretization of the wave equation, we get a system of ordinary differential equations (ODEs) which will be solved by the exponential integration factor method. The DG discretization leads to a relatively large number of degrees of freedom in comparison to other discretization methods. To efficiently evaluate the product of the matrix exponential and a vector, we perform Krylov subspace approximations.

Compared with the existing numerical method for wave equation, we believe our method has a number of other attractive properties. First, the generalized flux in our paper admits a wide variety of numerical flux choices which can ensure the energy conservation, including central flux and alternating flux. Second, we prove the optimal convergence in the $L^{2}$ norm in one space dimension. The optimal convergence for a variety of flux choices is observed experimentally. Finally, We use the Krylov exponential integration method which has a much better performance in computer time. The outline of the paper is as follows. In Section 2, we will present the LDG method with generalized alternating numerical flux for the first-order system transformed from the second-order wave equation. Then, the construction and analysis of the generalized Gauss-Radau projections are stated in Section 2.1. After that, we will give the stability and a priori error estimates in the $L^{2}$-norm in Sections 2.2 and 2.3. In Section 3, we then move to the time discretization of semidiscrete form by the matrix exponential method. In Section 4, we present some numerical experiments that confirm our theoretical analysis.

\section{The LDG Method}

In this paper, we consider the following one-dimensional second-order wave equations:

$$
u_{t t}+c^{2} u_{x x}=f(x, t), \quad-1 \leq x \leq 1,0 \leq t \leq T,
$$

with the initial condition

$$
u(x, 0)=g(x), \quad-1 \leq x \leq 1 u_{t}(x, 0)=h(x),
$$

and the boundary conditions

$$
\begin{aligned}
u(-1, t) & =u(1, t), \\
u_{x}(-1, t) & =u_{x}(1, t), \\
0 & \leq t \leq T,
\end{aligned}
$$

where the constant $c$ is the wave speed. Here, " $u(x, t)$ " represents the displacement of an elastic bar from its equilibrium position. The functions " $g$ " and " $h$ " in the initial condition are initial displacement and velocity. In our analysis, the functions $f \in L^{2}(0, T ;[-1,1])$. The initial condition $g \in H_{0}^{1}([-1,1])$ and $h \in L^{2}([-1,1])$.

We first introduce two auxiliary variables: $p=c u_{x}$ and $w=u_{t}$, and then we can rewrite (1) as a first-order system in space:

$$
\begin{aligned}
& w_{t}=c p_{x}+f, \\
& p_{t}=c w_{x} .
\end{aligned}
$$

Acoustic wave equations (4) and (5) are known as velocity-stress form, while equation (1) is known as displacement form. The two forms are equivalent through $p=c u_{x}$ and $w=u_{t}$.

The computational domain $\Omega=[-1,1]$ is divided into $J$ cells $I_{j}=\left[x_{j-(1 / 2)}, x_{j+(1 / 2)}\right]$, for $1 \leq j \leq J$, in which

$$
-1=x_{1 / 2}<x_{3 / 2}<\cdots<x_{J+(1 / 2)}=1,
$$

and we set $h_{j}=x_{j+(1 / 2)}-x_{j-(1 / 2)}$ and set $h_{\max }=\max _{j} h_{j}$ and $h_{\text {min }}=\min _{j} h_{j}$ as the length of the largest and the smallest subintervals, respectively. The associated finite element space is defined as piecewise polynomial space:

$$
V_{h}^{k}=\left\{v:\left.v\right|_{I_{j}} \in P^{k}\left(I_{j}\right), \quad j=1, \ldots, J\right\},
$$

where $P^{k}\left(I_{j}\right)$ denotes the set of all polynomials of degree up to $k$ on $I_{j}$. It is worth noting that polynomials in the space $V_{h}^{k}$ are allowed to have discontinuities across element boundaries.

Under the above conditions, the numerical solution in the element $I_{j}$ can be written as follows:

$$
\begin{aligned}
w_{h}(x, t) & =\sum_{m=0}^{k} w_{j, m}(t) v_{j}^{m}(x)=\sum_{m=0}^{k} w_{j, m}(t) P^{m}(\xi) \\
& =\mathbf{V}^{T}(x) \mathbf{W}_{j}(t),
\end{aligned}
$$

where $\mathbf{V}=\left(P_{0}(\xi), P_{1}(\xi), \ldots, P_{k}(\xi)\right)^{T}$ denotes the Legendre basis functions and $\mathbf{W}_{j}(t)=\left(w_{j, 0}, w_{j, 1}, \ldots, w_{j, k}\right)^{T}$ denote the degrees of freedom (DOF) in cell $I_{j}$. The global degrees of freedom in $\Omega$ are the set of $\mathbf{W}_{j}(t)$ as $\mathbf{W}=\left[\mathbf{W}_{1} ; \mathbf{W}_{2} ; \ldots, \mathbf{W}_{J}\right]$. $w_{j+(1 / 2)}^{+}$and $w_{j+(1 / 2)}^{-}$are values of $w$ at $x_{j+(1 / 2)}$ from the right cell $I_{j+1}$ and the left cell $I_{j}$, respectively:

$$
\begin{aligned}
& w_{j+(1 / 2)}^{+}=\lim _{\epsilon \longrightarrow 0+} w\left(x_{j+(1 / 2)}+\epsilon\right), \\
& w_{j+(1 / 2)}^{-}=\lim _{\epsilon \longrightarrow 0+} w\left(x_{j+(1 / 2)}-\epsilon\right),
\end{aligned}
$$

and at each element boundary point $x_{j+(1 / 2)}$, the jump term is denoted by 


$$
[v]_{j+(1 / 2)}=v_{j+(1 / 2)}^{+}-v_{j+(1 / 2)}^{-},
$$

and the weighted average is defined by

$$
v_{j+(1 / 2)}^{(\theta)}=\theta v_{j+(1 / 2)}^{-}+\widetilde{\theta} v_{j+(1 / 2)}^{+}, \quad j=1,2, \ldots, J-1,
$$

where $\tilde{\theta}=1-\theta$. In particular, when $\theta=1, P$ and $Q$ are Gauss-Radau projection $P^{-}$and $Q^{-}$, respectively.

The local discontinuous Galerkin method (LDG method) for (4) and (5) is then formulated as follows: find $w_{h}, p_{h} \in V_{h}^{k}$ so that for all test functions $v_{h}, q_{h} \in V_{h}^{k}$ and all $1 \leq j \leq J$ are satisfied:

$$
\begin{aligned}
\int_{I_{j}} w_{t} v \mathrm{~d} x= & -c \int_{I_{j}} p v_{x} \mathrm{~d} x+\left(c \widehat{p} v^{-}\right)_{j+(1 / 2)}-\left(c \widehat{p} v^{+}\right)_{j-(1 / 2)} \\
& +\int_{I_{j}} f v \mathrm{~d} x \\
\int_{I_{j}} p_{t} q \mathrm{~d} x= & -c \int_{I_{j}} w q_{x} \mathrm{~d} x+\left(c \widehat{w} q^{-}\right)_{j+(1 / 2)}-\left(c \widehat{w} q^{+}\right)_{j-(1 / 2)}
\end{aligned}
$$

where the numerical fluxes $\widehat{w}_{h}$ and $\widehat{p}_{h}$ are defined as follows:

$$
\begin{aligned}
& \left.\widehat{w}_{h}\right|_{j+(1 / 2)}=w_{h}^{(\widetilde{\theta})}, \\
& \left.\widehat{p}_{h}\right|_{j+(1 / 2)}=p_{h}^{(\theta)},
\end{aligned}
$$

for $j=0,1,2, \ldots, J$.

2.1. Generalized Gauss-Radau Projection. In order to obtain the optimal error estimates, we first introduce two forms of generalized Gauss-Radau projections: for any function $z$ that is smooth enough, the generalized Gauss-Radau projection of $z, P_{\theta} z$, is defined as the unique element in $P^{k}\left(I_{j}\right)$ satisfying

$$
\int_{I_{j}} P_{\theta} z v \mathrm{~d} x=\int_{I_{j}} z v \mathrm{~d} x, \quad \forall v \in P^{k-1}\left(I_{j}\right), \quad j=1,2, \ldots, J,
$$

$$
P_{\theta} z^{(\theta)}\left(x_{j+(1 / 2)}\right)=z^{(\theta)}\left(x_{j+(1 / 2)}\right), \quad j=1,2, \ldots, J-1 .
$$

Similarly, $Q_{\widetilde{\theta}} z$ is defined as

$$
\int_{I_{j}} Q_{\widetilde{\theta}} z q \mathrm{~d} x=\int_{I_{j}} z q \mathrm{~d} x, \quad \forall q \in P^{k-1}\left(I_{j}\right), \quad j=1,2, \ldots, J,
$$

$Q_{\theta} z^{(\widetilde{\theta})}\left(x_{j+(1 / 2)}\right)=z^{(\widetilde{\theta})}\left(x_{j+(1 / 2)}\right), \quad j=1,2, \ldots, J-1$.

Under the definition of the above projections, we can obtain the following lemma.
Lemma 1. Let projection $Z_{h}$ be either $P_{\theta}$ or $Q_{\widetilde{\theta}}$, and assume that the function $z$ is smooth enough. For $\theta \neq(1 / 2)$, the projection error $\eta=Z_{h} z-z$ satisfies

$$
\|\eta\|_{L^{2}\left(\Omega_{h}\right)}+h^{1 / 2}\|\eta\|_{L^{2}\left(\Gamma_{h}\right)} \leq C h^{k+1}\|z\|_{W_{\infty}^{k+1}\left(\Omega_{h}\right)},
$$

where $C>0$ is denoted a generic constant independent of $h$ and $z$.

The proof of this lemma is not repeated in this paper. For the concrete proof process, the reader is referred to the analysis of Lemma 3.2 in [14].

2.2. Stability Analysis. Now, we state the stability analysis of the above LDG method based on the generalized alternating numerical fluxes.

Theorem 1. The numerical solutions $w_{h}, p_{h}$ obtained by using the LDG method to solve wave equations (12) and (13) satisfy

$$
\left\|w_{h}\right\|^{2}+\left\|p_{h}\right\|^{2} \leq e^{c t} \int_{0}^{t}\|f\|^{2} \mathrm{~d} x .
$$

$$
\text { If } f=0,\left\|w_{h}\right\|^{2}+\left\|p_{h}\right\|^{2}=0 \text { is a constant for all time. }
$$

Proof. First, considering the definition of Gauss-Radau projections, (12) and (13) can be transformed into the following form:

$$
\begin{aligned}
& \int_{I_{j}}\left(w_{h}\right)_{t} v_{h} \mathrm{~d} x=-c \int_{I_{j}} p_{h} v_{h x} \mathrm{~d} x+\left.c \widehat{p}_{h}\left[v_{h}\right]\right|_{j-(1 / 2)} ^{j+(1 / 2)}+\int_{I_{j}} f v_{h} \mathrm{~d} x, \\
& \int_{I_{j}}\left(p_{h}\right)_{t} q_{h} \mathrm{~d} x=-c \int_{I_{j}} w_{h} q_{h x} \mathrm{~d} x+\left.c \widehat{w_{h}}\left[q_{h}\right]\right|_{j-(1 / 2)} ^{j+(1 / 2)} .
\end{aligned}
$$

Add the above two equations on the whole area, and for the numerical solutions, we have

$$
\begin{aligned}
& \int_{\Omega}\left(p_{h}\right)_{t} q_{h} \mathrm{~d} x+\int_{\Omega}\left(w_{h}\right)_{t} v_{h} \mathrm{~d} x+c B\left(w_{h}, v_{h}, p_{h}, q_{h}\right) \\
& =\int_{\Omega} f w_{h} \mathrm{~d} x,
\end{aligned}
$$

where

$$
\begin{aligned}
B\left(w_{h}, v_{h}, p_{h}, q_{h}\right)= & \sum_{j=1}^{J} \int_{I_{j}} w_{h} q_{h x} \mathrm{~d} x+\sum_{j=1}^{J} \int_{I_{j}} p_{h} v_{h x} \mathrm{~d} x \\
& +\sum_{j=1}^{J-1} \widehat{w_{h}}\left[q_{h}\right]_{j+(1 / 2)}+\sum_{j=1}^{J-1} \widehat{p_{h}}\left[v_{h}\right]_{j+(1 / 2)}
\end{aligned}
$$

Suppose $v_{h}=w_{h}, q_{h}=p_{h}$; then, taking them into (23), we have 


$$
\begin{aligned}
B\left(w_{h}, w_{h}, p_{h}, p_{h}\right)= & \sum_{j=1}^{J} \int_{I_{j}} w_{h} p_{h x} \mathrm{~d} x+\sum_{j=1}^{J} \int_{I_{j}} p_{h} w_{h x} \mathrm{~d} x \\
& +\sum_{j=1}^{J-1} \widehat{w}_{h}\left[p_{h}\right]_{j+(1 / 2)}+\sum_{j=1}^{J-1} \widehat{p}_{h}\left[w_{h}\right]_{j+(1 / 2)} .
\end{aligned}
$$

For the term $\sum_{j=1}^{J} \int_{I_{j}} w_{h} p_{h x} \mathrm{~d} x$, by using partial integration, we can obtain

$$
\begin{aligned}
\sum_{j=1}^{J} \int_{I_{j}} w_{h} p_{h x} \mathrm{~d} x= & -\sum_{j=1}^{J} \int_{I_{j}} p_{h} w_{h x} \mathrm{~d} x \\
& -\sum_{j=1}^{J}\left(p_{h}^{+} w_{h}^{+}-p_{h}^{-} w_{h}^{-}\right)_{j+(1 / 2)} .
\end{aligned}
$$

For $\sum_{j=1}^{J}\left(p_{h}^{+} w_{h}^{+}-p_{h}^{-} w_{h}^{-}\right)_{j+(1 / 2)}$, we have two ways of dealing with it as follows:

$$
\begin{aligned}
& \sum_{j=1}^{J}\left(p_{h}^{+} w_{h}^{+}-p_{h}^{-} w_{h}^{-}\right)_{j+(1 / 2)} \\
& \quad=\sum_{j=1}^{J}\left(p_{h}^{+} w_{h}^{+}-p_{h}^{+} w_{h}^{-}+p_{h}^{+} w_{h}^{-}-p_{h}^{-} w_{h}^{-}\right)_{j+(1 / 2)} \\
& \quad=\sum_{j=1}^{J}\left(p_{h}^{+}\left[w_{h}\right]+w_{h}^{-}\left[p_{h}\right]\right)_{j+(1 / 2)}, \\
& \sum_{j=1}^{J}\left(p_{h}^{+} w_{h}^{+}-p_{h}^{-} w_{h}^{-}\right)_{j+(1 / 2)} \\
& \quad=\sum_{j=1}^{J}\left(p_{h}^{+} w_{h}^{+}-w_{h}^{+} p_{h}^{-}+w_{h}^{+} p_{h}^{-}-p_{h}^{-} w_{h}^{-}\right)_{j+(1 / 2)} \\
& \quad=\sum_{j=1}^{J}\left(w_{h}^{+}\right)\left[p_{h}\right]+p_{h}^{-}\left[w_{h}\right]_{j+(1 / 2)} .
\end{aligned}
$$

Substituting (25)-(27) into (24), we can get $B_{h}=0$. The following equation can be set up by (22):

$$
\int_{\Omega}\left(w_{h}\right)_{t} w_{h} \mathrm{~d} x+\int_{\Omega}\left(p_{h}\right)_{t} p_{h} \mathrm{~d} x=\int_{\Omega} f w_{h} \mathrm{~d} x .
$$

It is obvious that

$$
\frac{1}{2} \frac{\mathrm{d}}{\mathrm{d} t}\left(\left\|w_{h}\right\|^{2}+\left\|p_{h}\right\|^{2}\right) \leq\|f\|\left\|w_{h}\right\| .
$$

Integrate both ends of the equation simultaneously:

$$
\left\|w_{h}\right\|^{2}+\left\|p_{h}\right\|^{2} \leq \int_{0}^{t}\|f\|^{2} \mathrm{~d} x+\int_{0}^{t}\left\|w_{h}\right\|^{2} \mathrm{~d} x .
$$

By the Gronwall inequality, we can obtain

$$
\left\|w_{h}\right\|^{2}+\left\|p_{h}\right\|^{2} \leq e^{c t} \int_{0}^{t}\|f\|^{2} \mathrm{~d} x .
$$

It is worth noting that $\left\|w_{h}\right\|^{2}+\left\|p_{h}\right\|^{2}=0$ can be established when $f=0$.
2.3. Error Estimate. In this section, we will discuss the error of the LDG method ((4) and (5)) with the periodic boundary.

Theorem 2. For the numerical solution $w_{h}$ of the wave equation obtained by (4) and (5) and the exact solution $w$, the following error estimates satisfy

$$
\left\|w(x, t)-w_{h}(x, t)\right\|^{2} \leq C h^{2 k+2},
$$

if $\theta \neq(1 / 2)$.

Proof. First, we will introduce new alternative notations to represent the error terms as follows:

$$
\begin{aligned}
& e_{w}=w-w_{h}=\left(w-P_{\theta} w\right)+\left(P_{\theta} w-w_{h}\right)=\eta_{w}-\varepsilon_{w}, \\
& e_{p}=p-p_{h}=\left(p-Q_{\theta} p\right)+\left(Q_{\theta} p-p_{h}\right)=\eta_{p}-\varepsilon_{p} .
\end{aligned}
$$

By Lemma 1, we can see that

$$
\left\|\eta_{w}\right\|_{L^{2}\left(\Omega_{h}\right)}+h^{1 / 2}\left\|\eta_{w}\right\|_{L^{2}\left(\Gamma_{h}\right)} \leq C h^{k+1}\|w\|_{k+1, \Omega_{h}} .
$$

So, in the following, we just need to prove that $\left\|\varepsilon_{w}\right\|_{L^{2}\left(\Omega_{h}\right)} \leq C h^{2 k+1}\|w\|_{k+1, \Omega_{h}}$.

For the exact solutions, it is true that

$$
\int_{\Omega} p_{t} q_{h} \mathrm{~d} x+\int_{\Omega} w_{t} v_{h} \mathrm{~d} x+c B\left(w, v_{h}, p, q_{h}\right)=\int_{\Omega} f v_{h} \mathrm{~d} x .
$$

Subtract (22) from (35) to get

$$
\begin{gathered}
\int_{\Omega}\left(\eta_{p}-\varepsilon_{p}\right)_{t} q_{h} \mathrm{~d} x+\int_{\Omega}\left(\eta_{w}-\varepsilon_{w}\right)_{t} v_{h} \mathrm{~d} x \\
+c B\left(\eta_{w}-\varepsilon_{w}, v_{h}, \eta_{p}-\varepsilon_{p}, q_{h}\right)=0 .
\end{gathered}
$$

Taking $v_{h}=\varepsilon_{w}, q_{h}=\varepsilon_{p}$ into (36),

$$
\begin{aligned}
& \int_{\Omega}\left(\varepsilon_{p}\right)_{t} \varepsilon_{p} \mathrm{~d} x+\int_{\Omega}\left(\varepsilon_{w}\right)_{t} \varepsilon_{w} \mathrm{~d} x+c B\left(\varepsilon_{w}, \varepsilon_{w}, \varepsilon_{p}, \varepsilon_{p}\right) \\
& \quad=\int_{\Omega}\left(\eta_{p}\right)_{t} \varepsilon_{p} \mathrm{~d} x+\int_{\Omega}\left(\eta_{w}\right)_{t} \varepsilon_{w} \mathrm{~d} x+c B\left(\eta_{w}, \varepsilon_{w}, \eta_{p}, \varepsilon_{p}\right) .
\end{aligned}
$$

According to the result of the stability analysis, the left hand side of (37) can be rewritten into

$$
\text { LHS }=\frac{1}{2} \frac{\mathrm{d}}{\mathrm{d} t}\left(\left\|\varepsilon_{w}\right\|^{2}+\left\|\varepsilon_{p}\right\|^{2}\right) .
$$

For the term $B\left(\eta_{w}, \varepsilon_{w}, \eta_{p}, \varepsilon_{p}\right)$ in the right hand side of (37), it can be derived that

$$
\begin{aligned}
B\left(\eta_{w}, \varepsilon_{w}, \eta_{p}, \varepsilon_{p}\right)= & \int_{\Omega} \eta_{p}\left(\varepsilon_{w}\right)_{x} \mathrm{~d} x+\int_{\Omega} \eta_{w}\left(\varepsilon_{p}\right)_{x} \mathrm{~d} x \\
& -\left.\widehat{\eta}_{p} \varepsilon_{w}\right|_{j-(1 / 2)} ^{j+(1 / 2)}-\left.\widehat{\eta_{w}} \varepsilon_{p}\right|_{j-(1 / 2)} ^{j+(1 / 2)} \\
= & \int_{\Omega} \eta_{p}\left(\varepsilon_{w}\right)_{x} \mathrm{~d} x+\int_{\Omega} \eta_{w}\left(\varepsilon_{p}\right)_{x} \mathrm{~d} x \\
& -\left(\varepsilon_{p}^{-} \eta_{w}^{(\widetilde{\theta})}\right)_{j+(1 / 2)}+\left(\varepsilon_{p}^{+} \eta_{w}^{(\widetilde{\theta})}\right)_{j-(1 / 2)} \\
& -\left(\varepsilon_{w}^{-} \eta_{p}^{(\theta)}\right)_{j+(1 / 2)}+\left(\varepsilon_{w}^{+} \eta_{p}^{(\theta)}\right)_{j-(1 / 2)} .
\end{aligned}
$$


TABLE 1: The $L^{2}$ spatial errors and order of convergence of $w_{h}$ for Example 1.

\begin{tabular}{|c|c|c|c|c|c|c|c|}
\hline & \multirow{2}{*}{$m$} & \multicolumn{2}{|c|}{$\theta=0.2$} & \multicolumn{2}{|c|}{$\theta=0.5$} & \multicolumn{2}{|c|}{$\theta=1.0$} \\
\hline & & Error & Order & Error & Order & Error & Order \\
\hline \multirow{4}{*}{$P^{2}$} & 6 & $2.15 E-1$ & - & $2.08 E-1$ & - & $3.08 E-1$ & - \\
\hline & 7 & $1.80 E-2$ & 3.58 & $1.72 E-2$ & 3.59 & $1.88 E-2$ & 4.03 \\
\hline & 8 & $3.99 E-3$ & 2.17 & $2.07 E-3$ & 3.05 & $2.22 E-3$ & 3.05 \\
\hline & 9 & $3.74 E-4$ & 3.41 & $2.59 E-4$ & 2.99 & $2.66 E-4$ & 3.09 \\
\hline \multirow{4}{*}{$P^{3}$} & 6 & $2.23 E-2$ & - & $2.44 E-2$ & - & $1.45 E-2$ & - \\
\hline & 7 & $1.00 E-3$ & 4.47 & $3.87 E-3$ & 2.96 & $8.94 E-4$ & 4.02 \\
\hline & 8 & $8.70 E-5$ & 3.52 & $4.96 E-4$ & 2.96 & $8.45 E-5$ & 3.40 \\
\hline & 9 & $2.92 E-6$ & 4.89 & $5.78 E-5$ & 3.10 & $4.84 E-6$ & 4.13 \\
\hline
\end{tabular}

Noticing the definition of Gauss-Radau projections (15)-(18), the term $\int_{\Omega} \eta_{w}\left(\varepsilon_{p}\right)_{x} \mathrm{~d} x=0$ in (39) since $\varepsilon_{p} \in P^{k}\left(I_{j}\right)$ and $\left(\varepsilon_{p}\right)_{x} \in P^{k-1}\left(I_{j}\right)$. Similarly, $\int_{\Omega} \eta_{p}\left(\varepsilon_{w}\right)_{x}$ $\mathrm{d} x=0,\left(\varepsilon_{p}^{-} \eta_{w}^{(\widetilde{\theta})}\right)_{j+(1 / 2)}=\left(\varepsilon_{p}^{+} \eta_{w}^{(\widetilde{\theta})}\right)_{j-(1 / 2)}$, and $\left(\varepsilon_{w}^{-} \eta_{p}^{\widetilde{(\theta)}}\right)_{j+(1 / 2)}=$ $\left(\varepsilon_{w}^{+} \eta_{p}^{(\tilde{\theta})}\right)_{j-(1 / 2)}$. In this case, we can get the conclusion that $B\left(\eta_{w}, \varepsilon_{w}, \eta_{p}, \varepsilon_{p}\right)=0$.

So the right hand side of (37) can be transformed into

$$
\text { RHS }=\int_{\Omega}\left(\eta_{p}\right)_{t} \varepsilon_{p} \mathrm{~d} x+\int_{\Omega}\left(\eta_{w}\right)_{t} \varepsilon_{w} \mathrm{~d} x .
$$

Now equation (37) is changed into

$\frac{1}{2} \frac{\mathrm{d}}{\mathrm{d} t}\left(\left\|\varepsilon_{w}\right\|^{2}+\left\|\varepsilon_{p}\right\|^{2}\right)=\int_{\Omega}\left(\eta_{p}\right)_{t} \varepsilon_{p} \mathrm{~d} x+\int_{\Omega}\left(\eta_{w}\right)_{t} \varepsilon_{w} \mathrm{~d} x$.

Considering the basic inequality, we can obtain that

$$
\begin{aligned}
& \int_{\Omega}\left(\eta_{p}\right)_{t} \varepsilon_{p} \mathrm{~d} x+\int_{\Omega}\left(\eta_{w}\right)_{t} \varepsilon_{w} \mathrm{~d} x \leq\left\|\left(\eta_{w}\right)_{t}\right\|^{2}+\left\|\varepsilon_{w}\right\|^{2} \\
& \quad+\left\|\left(\eta_{p}\right)_{t}\right\|^{2}+\left\|\varepsilon_{p}\right\|^{2} .
\end{aligned}
$$

According to Lemma 1, we can get

$$
\begin{gathered}
\left\|\left(\eta_{w}\right)_{t}\right\|^{2} \leq C h^{2 k+2}, \\
\left\|\left(\eta_{p}\right)_{t}\right\|^{2} \leq C h^{2 k+2} .
\end{gathered}
$$

Combining equation (41), we can get

$$
\frac{1}{2} \frac{\mathrm{d}}{\mathrm{d} t}\left(\left\|\varepsilon_{w}\right\|^{2}+\left\|\varepsilon_{p}\right\|^{2}\right) \leq C h^{2 k+2}+\left\|\varepsilon_{w}\right\|^{2}+\left\|\varepsilon_{p}\right\|^{2} .
$$

Assume that $\phi=\left\|\varepsilon_{w}\right\|^{2}+\left\|\varepsilon_{p}\right\|^{2}$; when the left and right ends are simultaneously multiplied by the integral factor $e^{-t}$, (44) can be converted into

$$
e^{-t} \frac{\mathrm{d} \phi}{\mathrm{d} t} \leq e^{-t} C h^{2 k+2}+e^{-t} \phi .
$$

For the integration of (45), we can get

$$
e^{-t} \phi \leq C h^{2 k+2} \int_{0}^{t} e^{-\tau} \mathrm{d} \tau,
$$

where $\phi \leq C h^{2 k+2} e^{T}$.

Finally, we can obtain that

$$
\left\|w-w_{h}\right\|^{2} \leq\left\|\eta_{w}\right\|^{2}+\left\|\varepsilon_{w}\right\|^{2} \leq C h^{2 k+2} .
$$

\section{Time Discretization: Krylov Implicit Integration Factor Method}

After the LDG discretization of (4) and (5) in space, a set of ordinary differential equations is obtained:

$$
\begin{aligned}
& \mathbf{M}_{1} \frac{\mathrm{d} \mathbf{w}}{\mathrm{d} t}=\mathbf{A}_{1} \mathbf{P}+\mathbf{F}, \\
& \mathbf{M}_{2} \frac{\mathrm{d} \mathbf{P}}{\mathrm{d} t}=\mathbf{A}_{2} \mathbf{W},
\end{aligned}
$$

where $\mathbf{U}$ and $\mathbf{P}$ are solution vectors containing the degrees of freedom of $u$ and $\mathbf{p}$. Because the mass matrices $\mathbf{M}_{1}$ and $\mathbf{M}_{2}$ are block diagonal, the mass matrices can be inverted easily to get

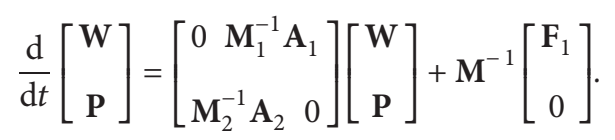

Set $\Phi=\left[\begin{array}{c}\mathbf{W} \\ \mathbf{P}\end{array}\right]$ and $\mathbf{F}=\mathbf{M}^{-1}\left[\begin{array}{c}\mathbf{F}_{1} \\ 0\end{array}\right], \mathbf{A}=\left[\begin{array}{ccc}0 & \mathbf{M}_{1}^{-1} \mathbf{A}_{1} \\ \mathbf{M}_{2}^{-1} & \mathbf{A}_{2} & 0\end{array}\right]$. Then, (49) can be rewritten into

$$
\frac{\mathrm{d}}{\mathrm{d} t} \Phi=\mathbf{A} \Phi+\mathbf{F} .
$$

Assume the final time is $t=T$ and let time step $\Delta t=T / N, t_{n}=n \Delta t, 0 \leq n \leq N$. We multiply (50) by the integration factor $e^{-\mathrm{A} t}$ and integrate over one time step from $t_{n}$ to $t_{n+1}$ to obtain

$$
\Phi_{n+1}=e^{\mathbf{A} \Delta t} \Phi_{n}+e^{\mathbf{A} \Delta t} \int_{0}^{\Delta t} e^{-\mathbf{A} \tau} \mathbf{F}(\tau) \mathrm{d} \tau .
$$

Using the trapezoidal integration to calculate the integral in (52), the second-order exponential integration factor formula is obtained:

$$
\Phi_{n+1}=e^{\mathrm{A} \Delta t}\left(\Phi_{n}+\frac{\Delta t}{2} \mathbf{F}_{n}\right)+\frac{\Delta t}{2} \mathbf{F}_{n+1} .
$$

It is noticed that if $f=0$, the exponential integration factor is exact. To construct such a scheme, we need to formulate an efficient algorithm for computing the products 
TABLE 2: The $L^{2}$ spatial errors and order of convergence of $p_{h}$ for Example 1.

\begin{tabular}{|c|c|c|c|c|c|c|c|}
\hline & \multirow{2}{*}{$m$} & \multicolumn{2}{|c|}{$\theta=0.2$} & \multicolumn{2}{|c|}{$\theta=0.5$} & \multicolumn{2}{|c|}{$\theta=1.0$} \\
\hline & & Error & Order & Error & Order & Error & Order \\
\hline \multirow{4}{*}{$P^{2}$} & 6 & $1.74 E-1$ & - & $2.08 E-1$ & - & $3.49 E-1$ & - \\
\hline & 7 & $1.98 E-2$ & 3.13 & $1.72 E-2$ & 3.59 & $4.06 E-2$ & 3.10 \\
\hline & 8 & $3.01 E-3$ & 2.72 & $2.07 E-3$ & 3.05 & $4.00 E-3$ & 3.34 \\
\hline & 9 & $3.73 E-4$ & 3.01 & $2.59 E-4$ & 2.99 & $3.59 E-4$ & 3.48 \\
\hline \multirow{4}{*}{$P^{3}$} & 6 & $2.25 E-2$ & - & $2.44 E-2$ & - & $1.25 E-2$ & - \\
\hline & 7 & $1.87 E-3$ & 3.58 & $3.87 E-3$ & 2.96 & $7.27 E-4$ & 4.11 \\
\hline & 8 & $1.33 E-4$ & 3.81 & $4.96 E-4$ & 2.96 & $6.10 E-5$ & 3.58 \\
\hline & 9 & $5.06 E-6$ & 4.72 & $5.78 E-5$ & 3.10 & $4.36 E-6$ & 4.16 \\
\hline
\end{tabular}

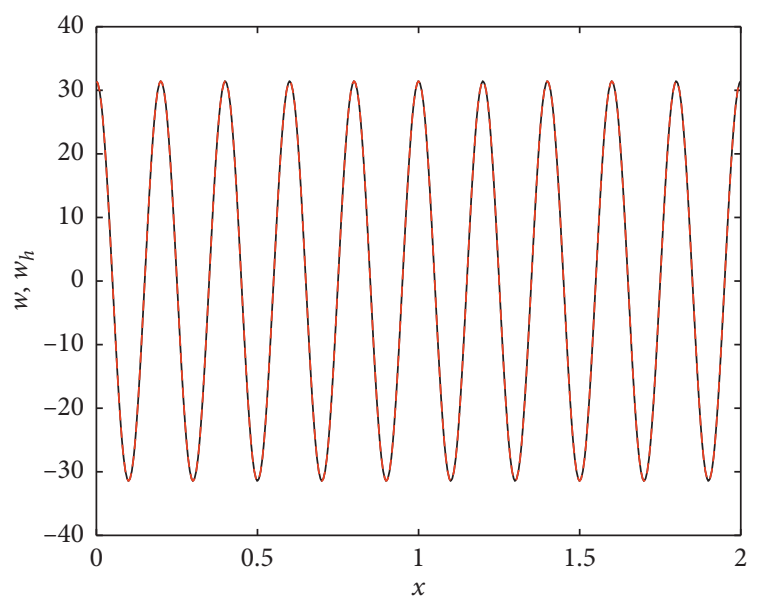

$-\begin{gathered}w \\ ---w_{h}\end{gathered}$

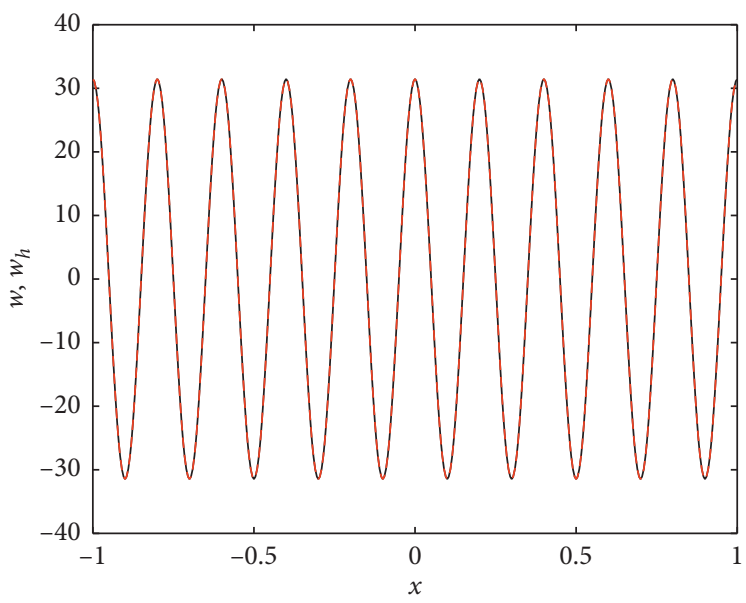

$-w$
$---w_{h}$

(a)

(b)

Figure 1: The exact solution $w$ (solid line) and LDG solution $w_{h}$ (dashed red dots) at $T=100$ by $P^{2}$ (a) and $P^{3}$ (b) for Example 1 .

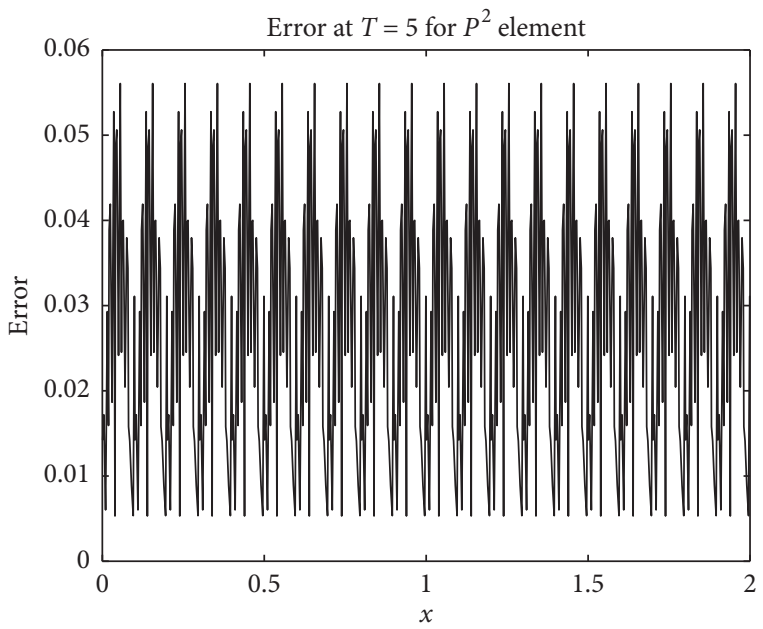

(a)

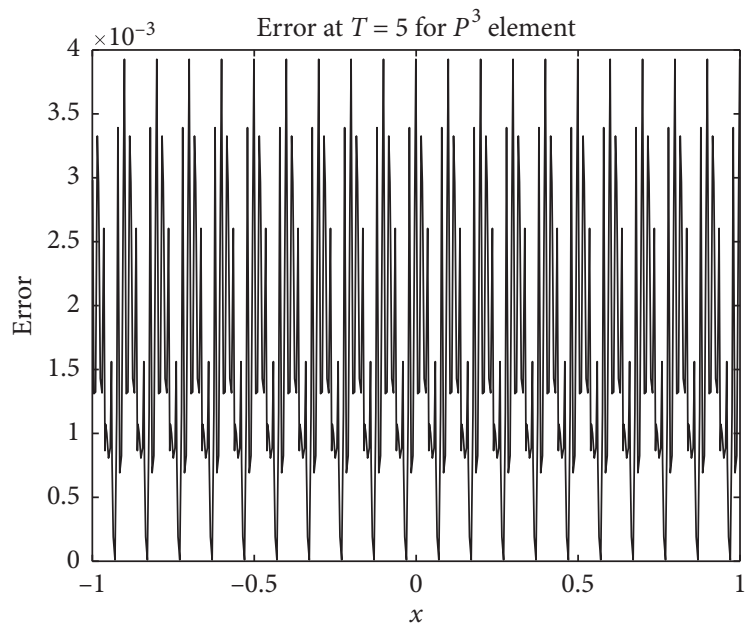

(b)

Figure 2: The error $\left\|w(x, t)-w_{h}(x, t)\right\|$ versus $x$ at $T=5$ by $P^{2}$ (a) and $P^{3}$ (b) for Example 1 . 


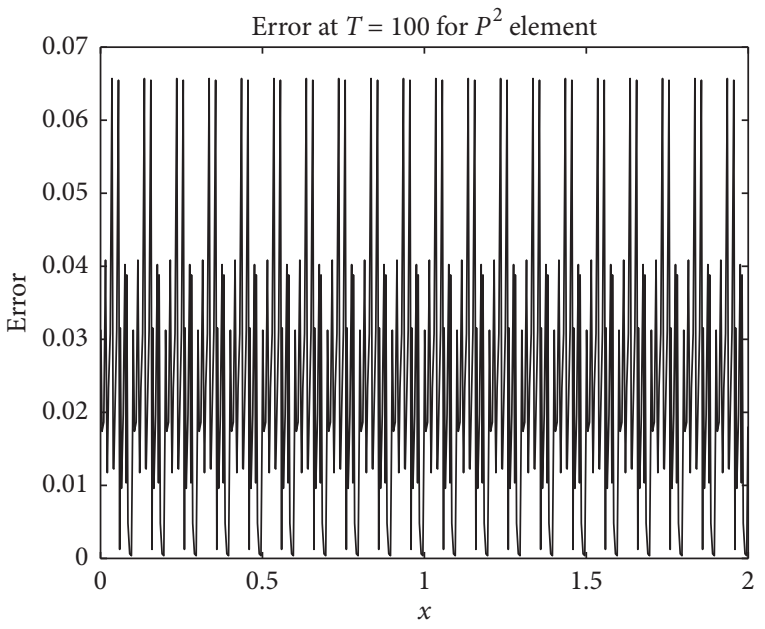

(a)

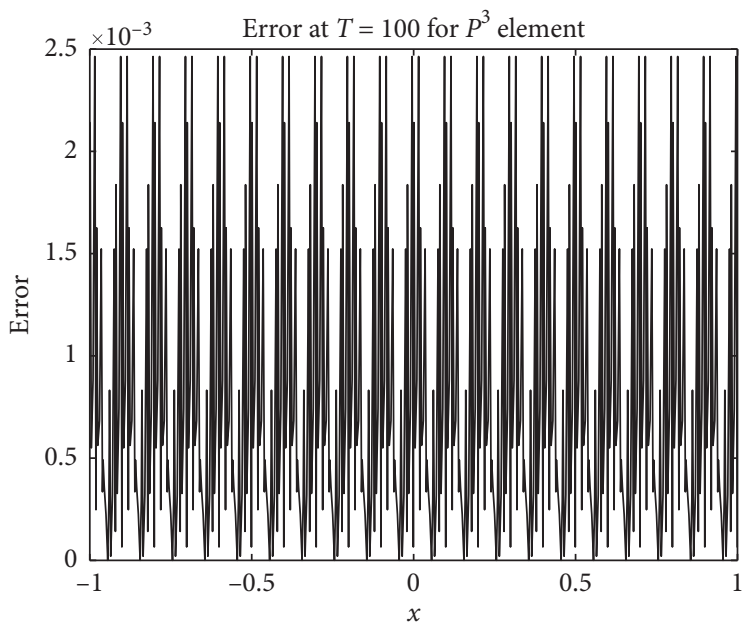

(b)

Figure 3: The error $\left\|w(x, t)-w_{h}(x, t)\right\|$ versus $x$ at $T=100$ by $P^{2}$ (a) and $P^{3}$ (b) for Example 1 .

of matrices $e^{\mathbf{A} \Delta t}$ and vector $\Phi_{n}$. In this paper, we accomplish the product by using Krylov subspace projections. The matrix $\mathbf{A}$ and vector $\Phi_{n}$ is projected on a Krylov subspace:

$$
K_{m}\left(A, \Phi_{n}\right)=\operatorname{span}\left\{\Phi_{n}, A \Phi_{n}, \ldots, A^{m-1} \Phi_{n}\right\} .
$$

The orthonormal basis $\left\{v_{1}, v_{2}, \ldots, v_{m}\right\}$ in the subspace $K_{m}\left(\mathbf{A}, \Phi_{n}\right)$ is constructed using an algorithm based on the Gram-Schmidt orthogonalization. A numerically preferable version of this algorithm is the Arnoldi modified Gram-Schmidt procedure. We refer the reader to the paper [17] for the detailed description of the Arnoldi algorithm. A Krylov subspace representation $H_{m}$ of the matrix $\mathbf{A}$ is constructed in the process of orthogonalization. Then, the obtained results are used to evaluate the approximation to $e^{\mathbf{A} \Delta t}$ in $K_{m}\left(\mathbf{A}, \Phi_{n}\right)$. In this section, we take $m=25$.

\section{Numerical Experiments}

In this section, the solutions of the wave equation will be investigated by using the proposed LDG method. The LDG method is carried out using the spaces $P^{k}$ with $k=2,3$ in (7). All experiments were carried out via Matlab on a PC with Intel Core i7 $1.8 \mathrm{GHz}$ processor and 16 GB RAM.

Example 1. We first consider the following homogeneous wave problem with the periodic boundary conditions:

$$
\begin{aligned}
u_{t t}-u_{x x} & =0, \quad x \in[-1,1], t \in[0, T], \\
u(x, 0) & =\sin (10 \pi x), \\
u_{t}(x, 0) & =10 \pi \cos (10 \pi x), \\
x & \in[-1,1], \\
u(-1, t) & =u(1, t), \\
u_{x}(-1, t) & =u_{x}(1, t) .
\end{aligned}
$$

The exact solution of this problem can be given by $u(x, t)=\sin (10 \pi(x+t))$.

We solve this problem using the LDG method on uniform meshes having $J=32,64,128$, and 256. The time step is chosen as $\Delta t=10^{-3}$. Tables 1 and 2 list the $L^{2}$ errors and corresponding convergence orders for $w_{h}$ and $p_{h}$. In each table, the parameter $\theta$ is taken as $\theta=0.2,0.5$, and 1.0. The tables show different convergence orders with different $\theta$. For $\theta \neq(1 / 2)$, the optimal accuracy of $(k+1)$-th order for $k=2,3$ is obtained. For $\theta=1 / 2$, the suboptimal accuracy of $k$-th order for $k=3$ and optimal $(k+1)$-th order with for $k=2$ can be observed. This coincides with Theorem 2 and shows the sharpness of theoretical results.

We present the true and LDG solution at time $T=100$ in Figure 1 with finite element spaces $P^{2}$ and $P^{3}$ and $J=100$. Figure 1 shows that the shape of the solution after long time integration is well preserved by using the LDG method. In Figures 2 and 3, we show the time evolution of the error curves of $w_{h}$ at time $T=1$ and $T=100$. These computations are also performed with finite element spaces $P^{2}$ and $P^{3}$ and $J=100$. The obtained results indicate that the $L^{2}$ errors do not grow in time and match well with the numerical results reported in [18]. The example shows that the proposed LDG scheme has very good dissipation and dispersion properties.

Example 2. We then consider the nonhomogeneous wave problem:

$$
u_{t t}-u_{x x}=f(x, t), \quad x \in[-1,1], t \in[0, T] .
$$

The exact solution of this problem is given by $u(x, t)=\sin (\pi x) e^{-0.1 t}$. The initial condition and $f(x, t)$ are determined by the exact solution.

We start by solving (55) on uniform meshes having $J=16,32,64$, and 128 . We choose the same parameters of $\theta$ as Example 1. The $L^{2}$ norm errors and corresponding 
TABLE 3: The $L^{2}$ spatial errors and order of convergence of $p_{h}$ for Example 2.

\begin{tabular}{|c|c|c|c|c|c|c|c|}
\hline & \multirow{2}{*}{$m$} & \multicolumn{2}{|c|}{$\theta=0.2$} & \multicolumn{2}{|c|}{$\theta=0.5$} & \multicolumn{2}{|c|}{$\theta=1.0$} \\
\hline & & Error & Order & Error & Order & Error & Order \\
\hline \multirow{4}{*}{$P^{2}$} & 6 & $1.19 E-3$ & - & $7.80 E-4$ & - & $1.67 E-3$ & - \\
\hline & 7 & $1.41 E-4$ & 3.07 & $9.59 E-5$ & 3.02 & $2.20 E-4$ & 2.92 \\
\hline & 8 & $1.71 E-5$ & 3.05 & $1.20 E-5$ & 3.00 & $1.95 E-5$ & 3.49 \\
\hline & 9 & $2.30 E-6$ & 2.89 & $1.52 E-6$ & 2.97 & $1.59 E-6$ & 3.60 \\
\hline \multirow{4}{*}{$P^{3}$} & 4 & $4.54 E-5$ & - & $1.78 E-4$ & - & $1.99 E-5$ & - \\
\hline & 5 & $3.37 E-6$ & 3.75 & $2.82 E-5$ & 2.66 & $1.17 E-6$ & 4.09 \\
\hline & 6 & $3.02 E-7$ & 3.48 & $3.60 E-6$ & 2.97 & $8.13 E-8$ & 3.85 \\
\hline & 7 & $2.79 E-8$ & 3.44 & $5.29 E-7$ & 2.77 & $4.69 E-9$ & 4.11 \\
\hline
\end{tabular}

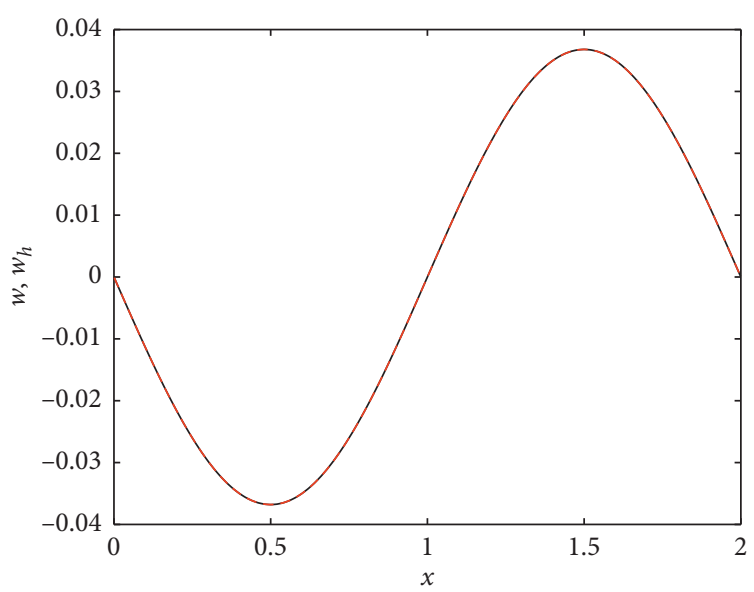

$-\begin{aligned} & w \\ & ---w_{h}\end{aligned}$

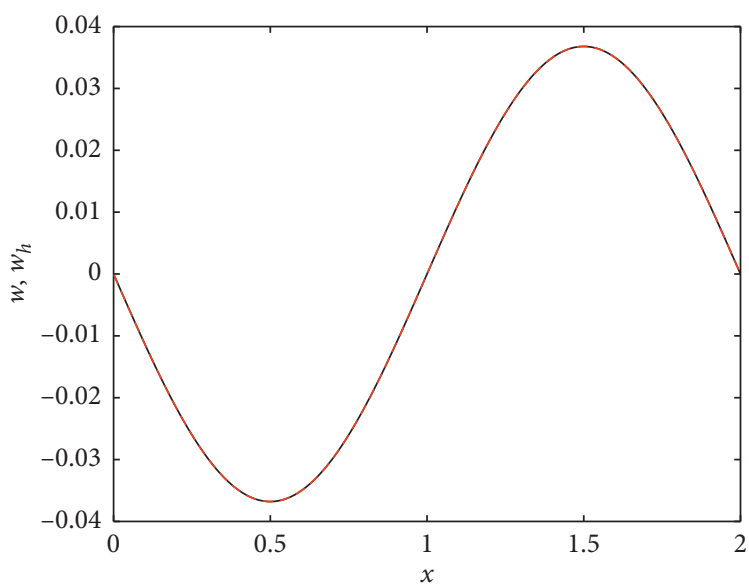

$-\begin{gathered}w \\ ---w_{h}\end{gathered}$

(a)

(b)

Figure 4: The exact solution $w$ (solid line) and LDG solution $w_{h}$ (dashed red dots) at $T=10$ by $P^{2}$ (a) and $P^{3}$ (b) for Example 2 .

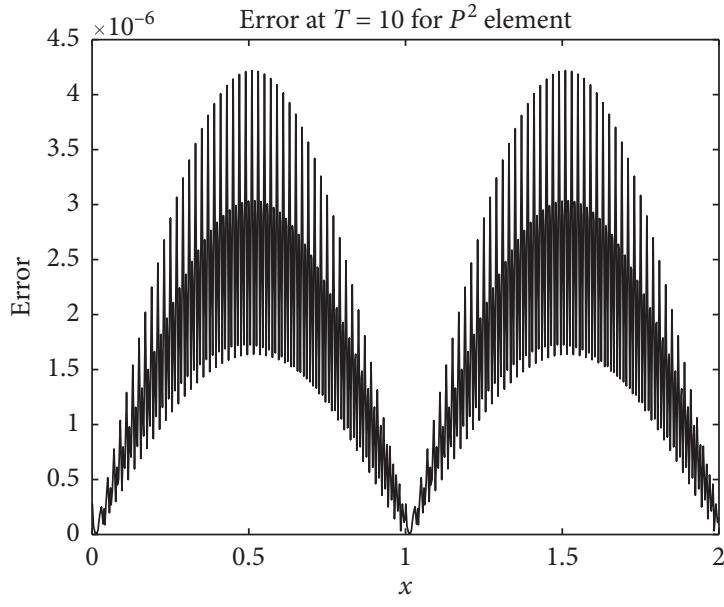

(a)

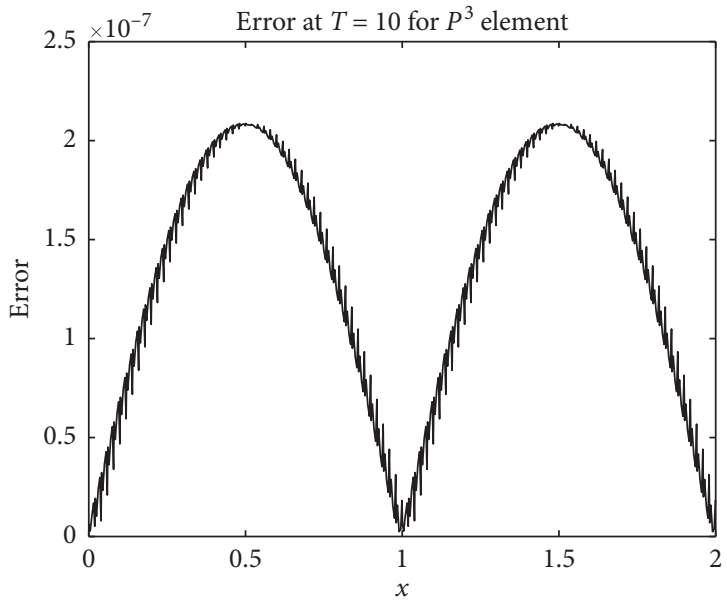

(b)

Figure 5: The error $\left\|w(x, t)-w_{h}(x, t)\right\|$ versus $x$ at $T=10$ by $P^{2}$ (a) and $P^{3}$ (b) for Example 2. 
convergence orders at time $T=1$ are shown in Table 3 for $p_{h}$. The error for $w_{h}$ is similar and we omit it. We can get the similar convergence order as in Example 1, which verifies the theoretical results in Section 2. Then, we show that the shape of the solution by LDG method will preserve after long time integration is well preserved. The shape of LDG solutions at $T=10$ with finite element spaces $P^{2}$ and $P^{3}$ is shown in Figure 4. The evolution of error curve is presented in Figure 5 with $P^{2}$ and $P^{3}$ elements.

\section{Summary}

In this paper, we have applied the LDG method with generalized alternating flux for solving the one-dimensional second-order wave equation with the periodic boundary conditions. The second-order wave equation is transformed into the first-order wave equation by introducing a variable. The first-order wave equation is then discretized by the LDG method which results in a stiff system of first-order ordinary differential equations. This system of ordinary differential equations is solved by the exponential matrix method analytically. Numerical results are compared with exact solutions at different times. The obtained results confirm that our LDG method is a powerful and reliable method for capturing the shock phenomenon. The methods extended to higher dimensional case will be our future work.

\section{Data Availability}

The numerical data used to support the findings of this study are included within the article.

\section{Conflicts of Interest}

The authors declare that there are no conflicts of interest regarding the publication of this paper.

\section{Acknowledgments}

This study was partially supported by the Foundation of LCP (no. 6142A0502020717) and Natural Science Foundation of Liaoning Province (grant no. 20180550996).

\section{References}

[1] A. Safjan and J. T. Oden, "High-order taylor-galerkin and adaptive h-p methods for second-order hyperbolic systems: application to elastodynamics," Computer Methods in Applied Mechanics Engineering, vol. 103, no. 1-2, pp. 187-230, 1993.

[2] G. Cohen, P. Joly, J. E. Roberts, and N. Tordjman, "Higher order triangular finite elements with mass lumping for the wave equation," Siam Journal on Numerical Analysis, vol. 38, no. 6, pp. 2047-2078, 2001.

[3] J. V. Lambers, "An explicit, stable, high-order spectral method for the wave equation based on block Gaussian quadrature," Iaeng International Journal of Applied Mathematics, vol. 38, no. 4, pp. 333-348, 2008.

[4] E. Zampieri and L. F. Pavarino, "An explicit second order spectral element method for acoustic waves," Advances in Computational Mathematics, vol. 25, no. 4, pp. 381-401, 2006.
[5] S. Mungkasi and S. G. Roberts, "On the best quantity reconstructions for a well balanced finite volume method used to solve the shallow water wave equations with a wet/dry interface," Anziam Journal, vol. 51, pp. C48-C65, 2010.

[6] B. Cockburn and C. W. Shu, "The local discontinuous galerkin finite element method for convection-di usion systems," SIAM Journal on Numerical Analysis, vol. 35, pp. 2440-2463, 1998.

[7] F. Bassi and S. Rebay, "High-order accurate discontinuous finite element solution of the $2 \mathrm{D}$ euler equations," Journal of Computational Physics, vol. 138, no. 2, pp. 251-285, 1997.

[8] S. Adjerid and H. Temimi, "A discontinuous galerkin method for the wave equation," Computer Methods in Applied Mechanics and Engineering, vol. 200, no. 5-8, pp. 837-849, 2011.

[9] M. Baccouch, "A local discontinuous galerkin method for the second-order wave equation," Computer Methods in Applied Mechanics and Engineering, vol. 209-212, no. 1, pp. 129-143, 2012.

[10] T. Lähivaara and T. Huttunen, "A non-uniform basis order for the discontinuous galerkin method of the acoustic and elastic wave equations," Applied Numerical Mathematics, vol. 61, no. 4, pp. 473-486, 2011.

[11] E. T. Chung and B. Engquist, "Optimal discontinuous galerkin methods for the acoustic wave equation in higher dimensions," Siam Journal on Numerical Analysis, vol. 47, no. 5, pp. 3820-3848, 2009.

[12] C.-S. Chou, C.-W. Shu, and Y. Xing, "Optimal energy conserving local discontinuous galerkin methods for secondorder wave equation in heterogeneous media," Journal of Computational Physics, vol. 272, no. 5, pp. 88-107, 2014.

[13] X. Meng, C.-W. Shu, and B. Wu, "Optimal error estimates for discontinuous galerkin methods based on upwind-biased fluxes for linear hyperbolic equations," Mathematics of Computation, vol. 85, no. 299, pp. 1225-1261, 2015.

[14] Y. Cheng, X. Meng, and Q. Zhang, "Application of generalized gausscradau projections for the local discontinuous galerkin method for linear convection-diffusion equations," Mathematics of Computation, vol. 86, no. 305, pp. 1233-1267, 2017.

[15] Y. Cheng and Q. Zhang, "Local analysis of the local discontinuous galerkin method with generalized alternating numerical flux for one-dimensional singularly perturbed problem," Journal of Scientific Computing, vol. 72, no. 2, pp. 792-819, 2017.

[16] R. Zhang, D. Wang, X. Yu, B. Chen, and Z. Wang, "The local discontinuous galerkin method with generalized alternating flux for solving burger's equation," Journal of Nonlinear Sciences and Applications, vol. 12, no. 5, pp. 300-313, 2019.

[17] S. Chen and Y.-T. Zhang, "Krylov implicit integration factor methods for spatial discretization on high dimensional unstructured meshes: application to discontinuous galerkin methods," Journal of Computational Physics, vol. 230, no. 11, pp. 4336-4352, 2011.

[18] M. Baccouch, "Superconvergence of the local discontinuous galerkin method applied to the one-dimensional second-order wave equation," Numerical Methods for Partial Differential Equations, vol. 30, no. 3, pp. 862-901, 2014. 